\title{
Macroparticle simulation studies of a proton beam halo experiment
}

\author{
J. Qiang \\ Accelerator and Fusion Research Division, Lawrence Berkeley National Laboratory, Berkeley, California 94720
}

P. L. Colestock, D. Gilpatrick, H.V. Smith, and T. P. Wangler

Los Alamos National Laboratory, Los Alamos, New Mexico 87545

M. E. Schulze

General Atomics, Los Alamos, New Mexico 87545

(Received 12 September 2002; published 13 December 2002)

\begin{abstract}
We report macroparticle simulations for comparison with measured results from a proton beam halo experiment in a 52-quadrupole periodic-focusing channel. An important issue is that the input phasespace distribution is not experimentally known. Three different initial distributions with different shapes predict different beam profiles in the transport system. Simulations have been fairly successful in reproducing the core of the measured matched-beam profiles and the trend of emittance growth as a function of the mismatch factor, but underestimate the growth rate of halo and emittance for mismatched beams. In this study, we find that knowledge of the Courant-Snyder parameters and emittances of the input beam is not sufficient for reliable prediction of the halo. Input distributions with greater population in the tails produce larger rates of emittance growth, a result that is qualitatively consistent with the particle-core model of halo formation in mismatched beams.
\end{abstract}

DOI: 10.1103/PhysRevSTAB.5.124201

PACS numbers: 29.27.Bd, 52.65.Rr

\section{INTRODUCTION}

The macroparticle simulation method is widely used in modern accelerator design and beam physics studies [1-5]. The method provides a quantitative model of the time evolution of charged-particle bunches in accelerators. It includes the boundary conditions, the physics associated with external focusing and acceleration, and space-charge forces from intraparticle Coulomb interactions within the bunch. With increasing beam intensity, the effects of space-charge forces become more important. The self-consistent treatment of space-charge forces in macroparticle simulations is necessary for describing quantitatively the beam dynamics. To ensure that macroparticle simulation codes include the most important physics effects, comparisons with experimental measurements are necessary. Such comparisons can test the assumptions and approximations used in the codes. More benchmarks against experimental data would further increase our confidence in the use of the simulation codes for real machine design.

In this paper, we present comparisons of simulations using the code IMPACT [4] with experimental measurements of the beam profiles including beam halo in a highcurrent proton beam. The measurements were made in a new 52-quadrupole beam-transport channel using a 6.7- $\mathrm{MeV}$ proton beam at the Low-Energy Demonstration Accelerator (LEDA) facility at Los Alamos National Laboratory [6,7]. A major goal of the experiment was to validate the beam-dynamics simulations of beam halo, using simulation codes such as IMPACT. Of particular importance was the validation of the space-charge routine. A three-dimensional space-charge model is used here because the proton beam at the entrance of the transport channel has been bunched in the radio frequency quadrupole (RFQ). The space-charge forces are three-dimensional forces instead of two-dimensional forces.

In addition to the simulation work reported in this paper, a study was recently completed [8] to analyze earlier measurements from quadrupole scans in the LEDA high-energy beam-transport system that were made before the 52-quadrupole channel was installed. That work involved a combination of macroparticle simulations and least-squares fitting to measured beam profiles, where the objective was to characterize the nominal $100-\mathrm{mA}$ beam. The present paper focuses instead on a simulation study of beam halo formation in the new 52quadrupole lattice. Future simulation work will include an attempt to apply the beam-characterization results from Ref. [8] to the more recent mismatched-beam measurements in the 52-quadrupole lattice.

The paper is divided into the following three sections. The macroparticle simulation model is described in Sec. II, a comparison of the simulation results and experimental measurements is given in Sec. III, and the conclusions are presented in Sec. IV.

\section{MACROPARTICLE SIMULATION MODEL}

Charged-particle dynamics in an accelerator can be described by the Vlasov equation:

$$
\frac{\partial f}{\partial t}+\dot{\mathbf{r}} \cdot \frac{\partial f}{\partial \mathbf{r}}+\dot{\mathbf{p}} \cdot \frac{\partial f}{\partial \mathbf{p}}=0,
$$

where $f$ denotes the particle distribution function, $\mathbf{r}$ is the 
spatial position, and $\mathbf{p}$ is the momentum with $\dot{\mathbf{p}}=\mathbf{F}$. The force $\mathbf{F}$ includes the contributions from both the external fields $\left(F_{\text {ext }}\right)$ and the space-charge force $F_{\text {sc }}$. The spacecharge force in this equation is a mean-field approximation of the $N$-body microparticle Coulomb force. In the moving frame, the space-charge force can be obtained from the solution of Poisson's equation

$$
\nabla^{2} \phi(\mathbf{r})=-\frac{\rho(\mathbf{r})}{\epsilon_{0}}
$$

and

$$
\mathbf{F}_{\mathrm{sc}}=-q \nabla \phi,
$$

where $\phi$ is the electrostatic potential in the moving frame, $\rho$ is the particle spatial charge density, and $\epsilon_{0}$ is the vacuum permittivity. The charge density can be calculated from the distribution function $f$ by

$$
\rho(\mathbf{r})=\int \mathbf{d}^{3} \mathbf{p} f(\mathbf{r}, \mathbf{p}) .
$$

The Poisson-Vlasov equations can be solved using the particle-in-cell approach in macroparticle simulation. Here, macroparticles are generated with the same charge-to-mass ratio as the real particles in the bunch. Using $z$ as the independent variable, the equations of motion are [9]

$$
\begin{gathered}
x^{\prime}=\frac{p_{x}}{p_{z}}, \\
y^{\prime}=\frac{p_{y}}{p_{z}}, \\
\psi^{\prime}=\frac{\frac{\omega}{c} \gamma}{p_{z}}-\frac{\frac{\omega}{c}}{\beta_{0}}, \\
p_{x}^{\prime}=\frac{q}{m_{0} c p_{z}}\left(\frac{\gamma}{c} \mathbf{E}+\mathbf{p} \times \mathbf{B}\right)_{x} \\
p_{y}^{\prime}=\frac{q}{m_{0} c p_{z}}\left(\frac{\gamma}{c} \mathbf{E}+\mathbf{p} \times \mathbf{B}\right)_{y} \\
p_{t}^{\prime}=\frac{q}{m_{0} c^{2}} E_{z 0}-\frac{q}{m_{0} c^{2} p_{z}} \mathbf{p} \cdot \mathbf{E},
\end{gathered}
$$

where $x$ and $y$ are transverse spatial coordinates, $p_{x}, p_{y}$, and $p_{z}$ are normalized momenta $\gamma \beta_{x}, \gamma \beta_{y}$, and $\gamma \beta_{z}, \psi$ is the phase relative to the reference particle defined by $\psi=$ $\omega\left(t-t_{g}\right), \omega$ is the assumed $\mathrm{rf}$ frequency, $t_{g}$ is the flight time of the reference particle, $p_{t}$ is the normalized energy deviation with respect to the reference particle, $p_{t}=$ $\gamma_{g}-\gamma$, where $\gamma_{g}$ is the $\gamma$ of the reference particle, $\gamma=$ $1 / \sqrt{1-\beta^{2}}, \beta_{i}=\frac{v_{i}}{c}$ with $i=x, y, z$. The speed of light is $c$, and $m_{0}$ is the rest mass of the particle. The electric field
$\mathbf{E}$ and magnetic field $\mathbf{B}$ include the contributions from external focusing and accelerating fields and the mean field of intraparticle Coulomb interactions. The trajectory of the reference particle on the axis of the accelerator can be determined from the following:

$$
\begin{gathered}
t_{g}^{\prime}=\frac{1}{\beta_{0} c}, \\
\gamma_{g}^{\prime}=\frac{q}{m_{0} c^{2}} E_{z 0}(z, t),
\end{gathered}
$$

where $E_{z 0}$ is the on-axis external electrical field, and $\beta_{0}=\sqrt{1-1 / \gamma_{g}^{2}}$.

The equations of motion for the macroparticles are integrated using a second-order leapfrog algorithm. The particles are first advanced in space by a half step using their velocities to advance the transverse coordinates. Then the particles are deposited onto a three-dimensional spatial grid to obtain the charge-density distribution. Since we use $z$ as the independent variable, the particles at a given longitudinal location have to be transformed back to the distribution at a fixed time before deposition onto the grid. A detailed discussion of the transformation can be found in the paper by Qiang et al. [9]. Poisson's equation is solved in cylindrical coordinates with perfect conducting wall boundary conditions transversely and a periodic boundary condition longitudinally. Fields on the grid are calculated from the potential using a central finite-difference scheme. The fields on the grid are reinterpolated back to the particles to obtain the total spacecharge forces on the particles. The particles are then advanced in momentum space by one step using both the external fields and the space-charge forces. Here, the space-charge forces include the contributions from both the electric field and the magnetic field of the beam. These fields are obtained from the Lorentz transformation of the electrostatic field in the beam frame to the laboratory frame. Finally, the particles are advanced another half step spatially using the updated velocities to complete a full step.

\section{SIMULATION RESULTS OF LEDA EXPERIMENT}

The LEDA facility consists of a 75-keV dc injector, a low-energy beam-transport (LEBT) system, and a RFQ, which accelerates the proton beam to $6.7 \mathrm{MeV}$. A schematic plot of the LEDA beam halo experiment transport system, which follows the RFQ, is given in Fig. 1 [10]. The transport system consists of 52 magnetic quadrupoles with alternating polarization to provide transverse strong focusing. The lattice is spatially periodic with a focusing period length of $42.0 \mathrm{~cm}$. The first four quadrupole gradients are independently adjustable to match the beam or to produce mismatches to excite the breathing mode or the quadrupole mode. The transverse beam 
52 quadrupole FODO lattice

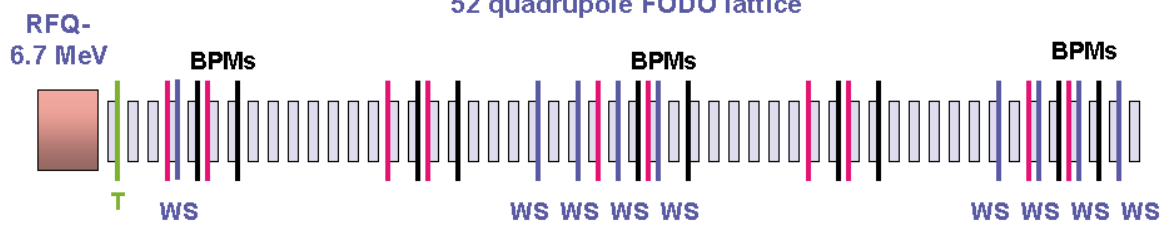

52 Quadrupoles

10 Steering Magnets

9 Wire Scanners

1 Toroid

10 Beam Position Monitors

FIG. 1. (Color) A schematic plot of the LEDA beam halo experiment transport system.

profiles were measured using beam profile scanners. The scanners are located in the middle of the drift space after quadrupoles 4, 20, 22, 24, 26, 45, 47, 49, and 51 . Besides quadrupoles and scanners, there are also ten beam-position monitors to determine beam-centroid positions, ten beam-steering magnets, and a beam toroid for beam-current measurements.

The output beam energy from the LEDA $350-\mathrm{MHz}$ RFQ is $6.7 \mathrm{MeV}$ and the beam current was varied from 16 to $100 \mathrm{~mA}$. In this paper we report on the $75-\mathrm{mA}$ results, for which the Courant-Snyder ellipse parameters and emittances for the distribution at the entrance of the transport system, calculated from the matched rms size measurements at first scanner cluster (20-26) are $\alpha_{x}=$ $1.17, \beta_{x}=0.255 \mathrm{~m} / \mathrm{rad}, \epsilon_{x}=2.93 \mathrm{~mm} \mathrm{mrad}$ (unnormalized $\mathrm{rms}), \alpha_{y}=-1.64, \beta_{y}=0.548 \mathrm{~m} / \mathrm{rad}$, and $\epsilon_{y}=$ $3.23 \mathrm{~mm} \mathrm{mrad}$ (unnormalized rms). The longitudinal parameters, determined from a multiparticle simulation through the RFQ are $\alpha_{z}=0.187, \beta_{z}=0.236^{\circ} / \mathrm{KeV}$, and $\epsilon_{z}=171.5^{\circ} \mathrm{KeV}$ (rms).

The lack of detailed knowledge of the initial distribution in phase space is an important issue. Our approach has been to generate three different initial distributions for comparison with measurements. These three initial distributions are 6D Waterbag, 6D Gaussian, and a distribution generated by a simulation through the LEBT and RFQ, starting at the plasma surface at the exit of the ion source. This distribution is called the LEBT/RFQ distribution, and should be a more realistic description of the beam at the entrance of the transport channel than the other two distributions. However, the simulation using the LEBT/RFQ initial distribution does not reproduce the Courant-Snyder parameters listed above and underestimates the matched rms sizes in the transport channel by about $30 \%$. Therefore, we have adjusted the particle coordinates from the LEBT/RFQ distribution to have the same Courant-Snyder parameters and emittances as listed above. In the rescaling process, we have defined in each plane of phase space, i.e., $x-p_{x}, y-p_{y}$, and phase-energy deviation, the quantities $f_{\epsilon}=\epsilon^{\text {given }} / \epsilon^{\text {old }}$, $r_{1}=\sqrt{\gamma^{\text {old }} / \gamma^{\text {given }}}, \quad r_{2}=1 / r_{1}, \quad$ and $\quad r_{3}=\left(\alpha^{\text {old }}-\right.$

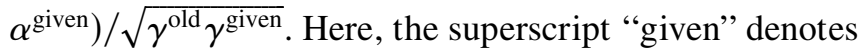
the quantity specified from the measurements, and "old" denotes the quantity before rescaling. The new coordinates in phase space after rescaling are given as

$$
\begin{gathered}
x^{t m p}=x^{\text {old }} f_{\epsilon}, \\
p_{x}^{t m p}=p_{x}^{\text {old }} f_{\epsilon}, \\
x=x^{t m p} r_{1}+p_{x}^{t m p} r_{3}, \\
p_{x}=p_{x}^{t m p} r_{2} .
\end{gathered}
$$

After the rescaling, the LEBT/RFQ distribution has the same Courant-Snyder parameters and emittances as in the Waterbag and Gaussian distributions. The transverse phase-space plots of these distributions are given in Fig. 2. As we progress from the Waterbag to the Gaussian to the LEBT/RFQ distribution, we note an increasing input beam halo.

Using these three initial distributions with a $75-\mathrm{mA}$ input beam current, we have simulated the beam transported through the matched LEDA experiment. We have used about $2.8 \times 10^{6}$ macroparticles per bunch with a computation grid of $65 \times 65 \times 129$. The rms beam size at the center of each drift space as a function of distance is given in Fig. 3 together with the experimental data. We see that all three distributions predict a nearly matched transverse $\mathrm{rms}$ beam size. The Waterbag distribution generates the best matched solution with a nearly uniform rms size through the channel. The rms beam size from the simulation using the LEBT/RFQ distribution fluctuates by about $10 \%$ from the average value. The matched rms sizes from measurements at nine locations in the transport channel show similar fluctuations. Besides the rms sizes, the LEDA experiment also measured the projected density distribution, i.e., beam profiles in $x$ and $y$ projections at nine locations along the transport channel.

Figures 4-6 give a comparison of density profiles from the simulations and from the measurements at the middle 

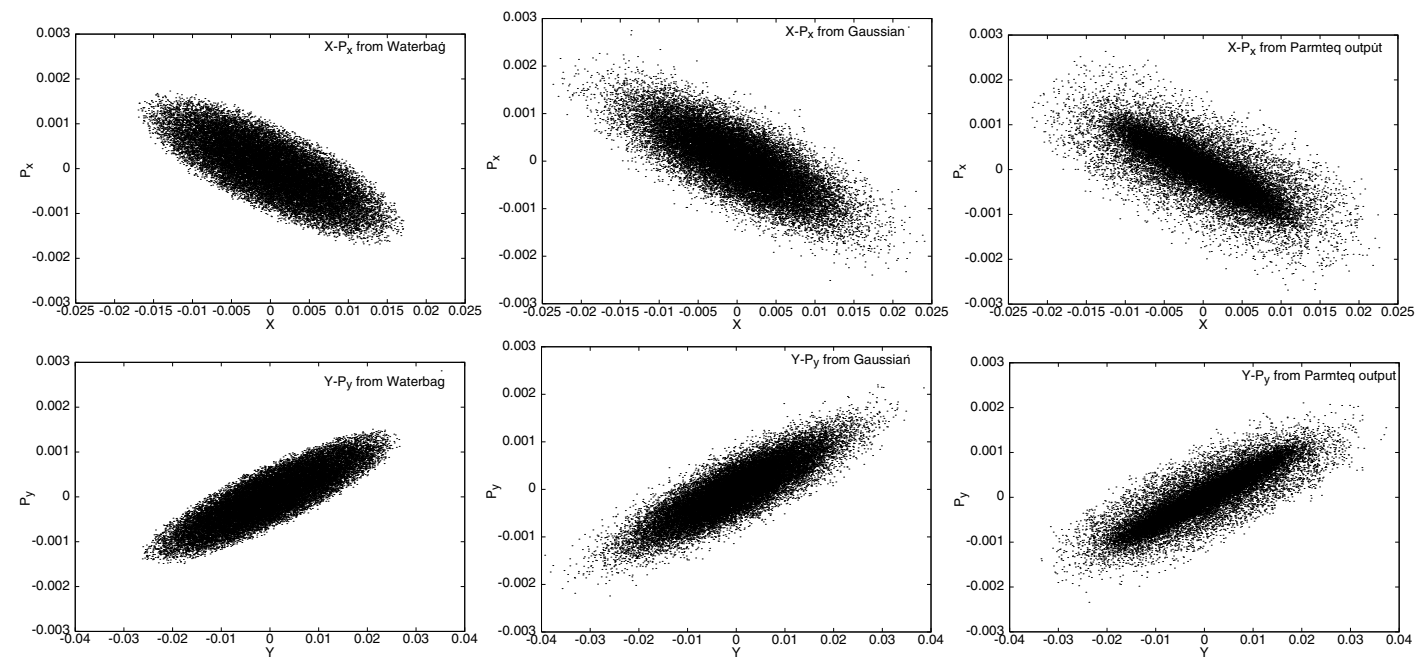

FIG. 2. Transverse phase-space projections from Waterbag, Gaussian, and the LEBT/RFQ distributions.

of the drift spaces where the scanners are located. Here, all the profiles from theory are generated from a selfconsistent macroparticle simulation including spacecharge forces, using the three initial distributions. There is no least-squares fitting of simulations to data in Figs. 4-6; the only adjustments made to the simulations are a shift of the coordinates to bring the centroids into agreement with measurements, and a normalization multiplier applied to all the simulation profiles to bring the integrated densities into agreement. Figure 4 shows the comparison between the measurements and the simulation using the Waterbag initial distribution. We see that at most locations, the density profiles from simulation do not reproduce the tails observed in the measured profiles, while the density profiles near the center from the simulation are broader than the measurements.

Figure 5 shows the comparison between the measurements and the simulation using the Gaussian initial distribution. Compared with the density profiles produced using the initial Waterbag distribution, the agreement between the simulation results and the measured beam profiles has improved. Figure 6 shows the comparison between the measurements and the simulation using the LEBT/RFQ initial distribution. In Fig. 6, we see that the density profiles predicted using this initial distribution agree best with the measured profiles especially near the peak.

In the LEDA experiment, the first four matching quadrupole magnets were also adjusted to produce rms mismatch. Figures 7-9 show the comparison of these density profiles from simulations, using the same three initial distributions, with measurements for a breathing mode oscillation with a mismatch factor $\mu=1.5$, where the mismatch factor $\mu$ is defined as the ratio of the initial rms size of the mismatched beam to that of the matched beam. In practice the matching quadrupole gradient solutions were computed using the code TRACE3D [11] to establish the desired rms sizes at scanner 20. By comparison with

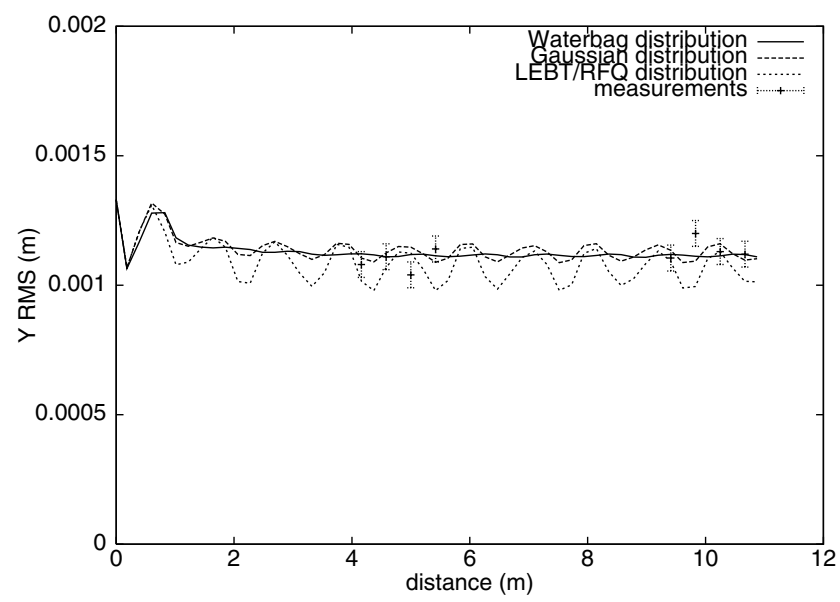

FIG. 3. $\quad X$ and $y$ rms size at the center of the drift space as a function of distance at $75 \mathrm{~mA}$. 

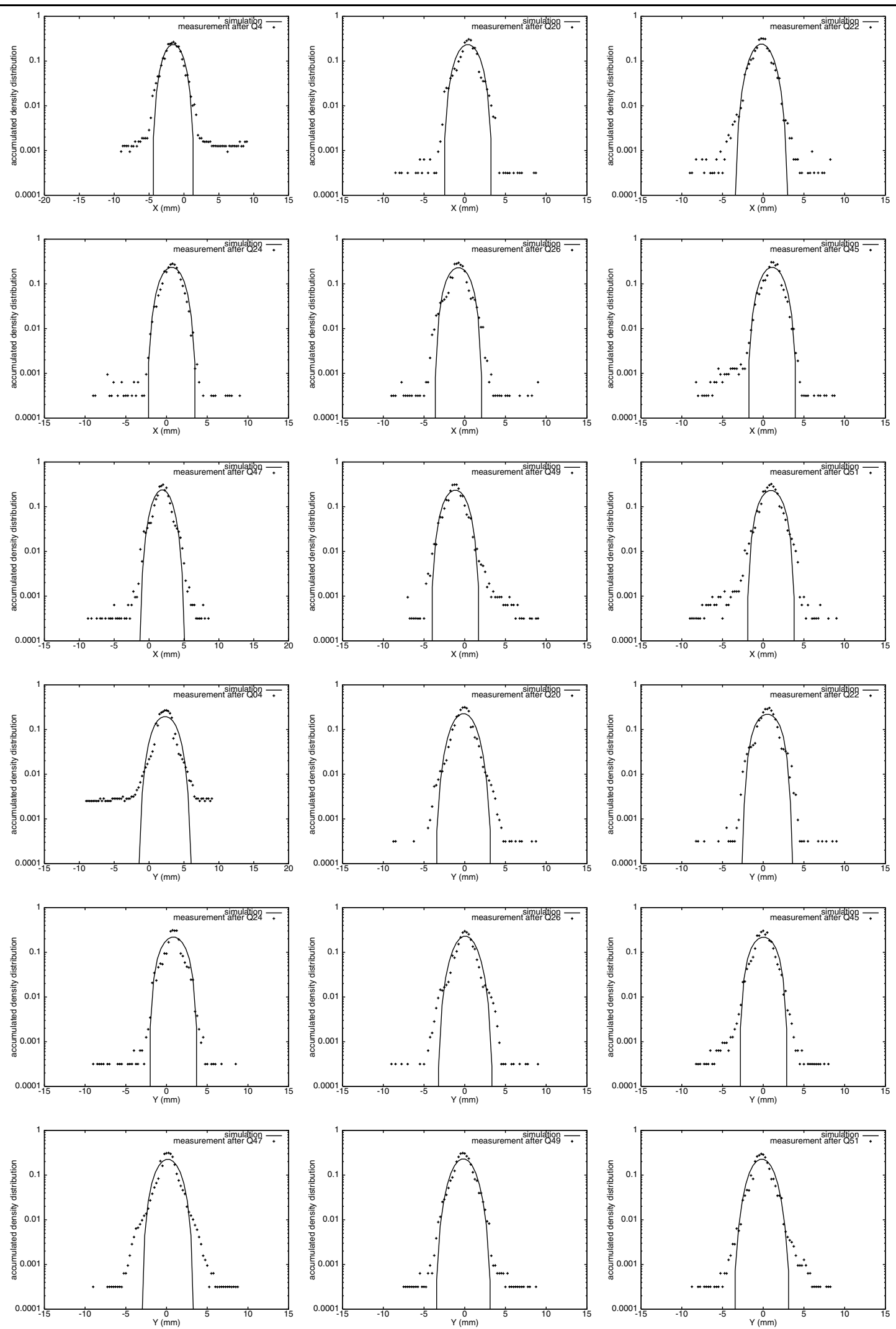

FIG. 4. $X$ and $y$ density distribution from simulation using initial Waterbag distribution and from measurements at $75 \mathrm{~mA}$ matched case. The first nine plots correspond to the nine $x$ profiles and the second nine plots correspond to the nine $y$ profiles. 

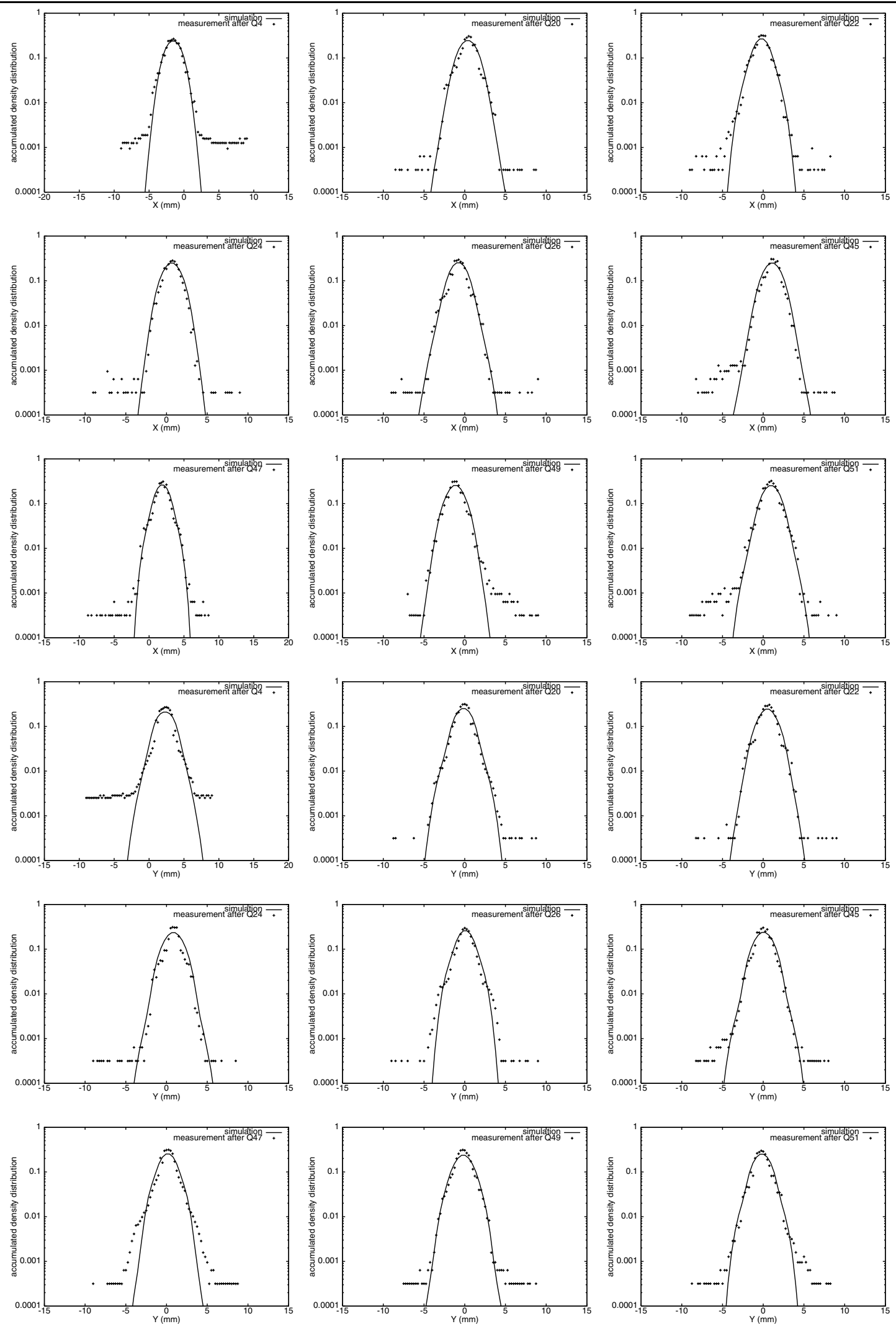

FIG. 5. $X$ and $y$ density distribution from simulation using initial Gaussian distribution and from measurements at $75 \mathrm{~mA}$ matched case. The first nine plots correspond to the nine $x$ profiles and the second nine plots correspond to the nine $y$ profiles. 

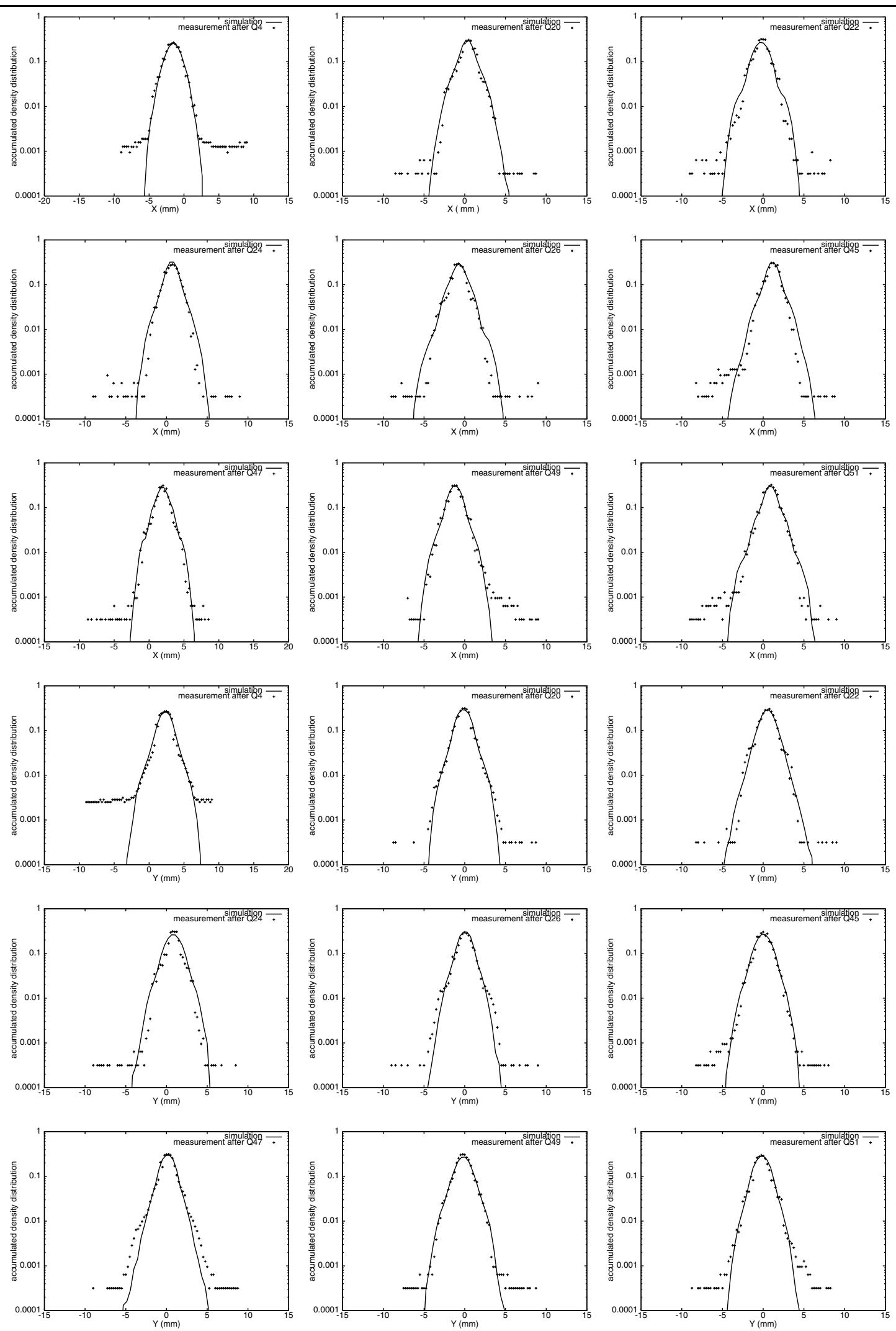

FIG. 6. $X$ and $y$ density distribution from simulation using initial scaled LEBT/RFQ distribution and from measurements at $75 \mathrm{~mA}$ matched case. The first nine plots correspond to the nine $x$ profiles and the second nine plots correspond to the nine $y$ profiles. 

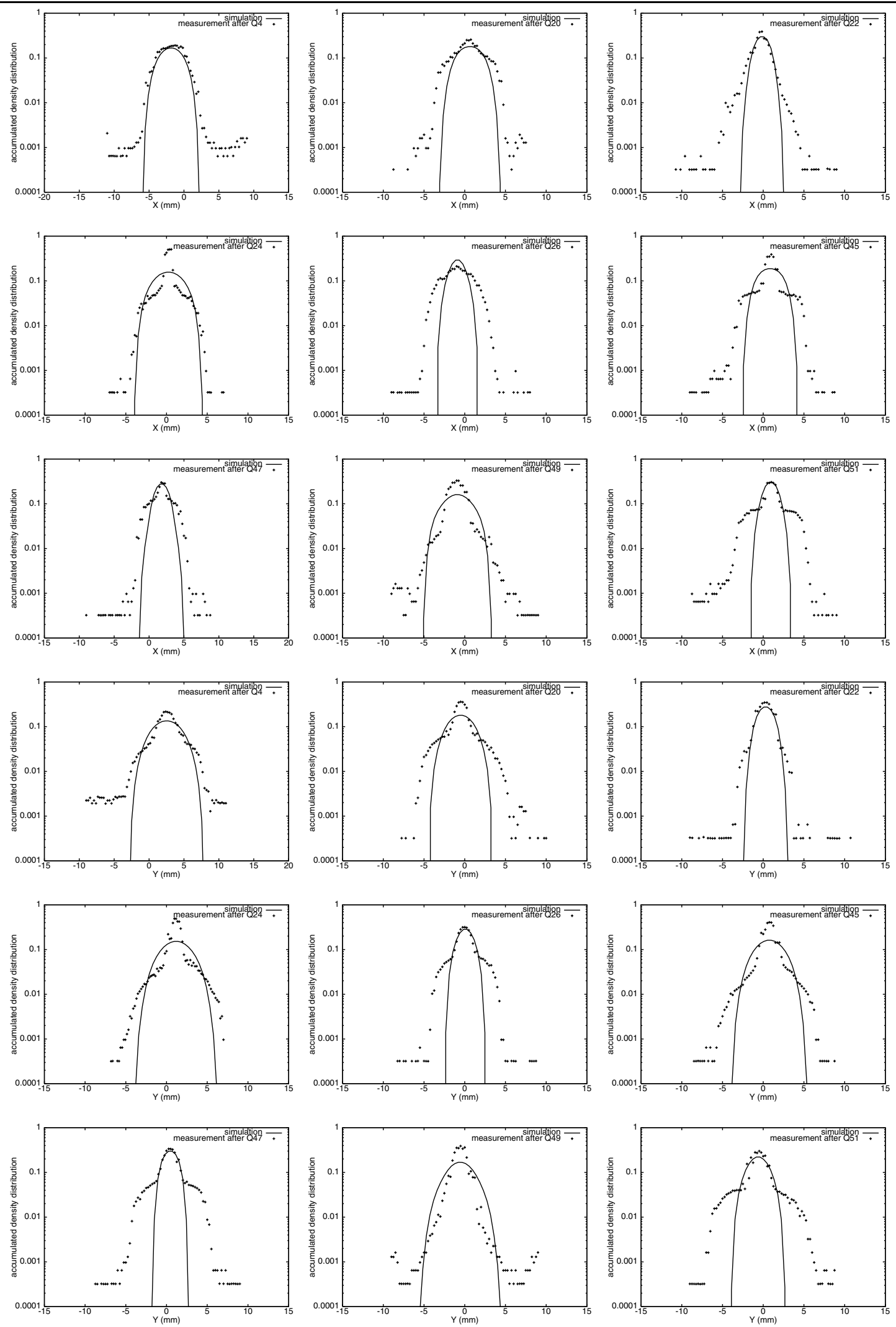

FIG. 7. $X$ and $y$ density distribution from simulation using initial Waterbag distribution and from measurements at $75 \mathrm{~mA}$ and mismatch factor of 1.5. The first nine plots correspond to the nine $x$ profiles and the second nine plots correspond to the nine $y$ profiles. 

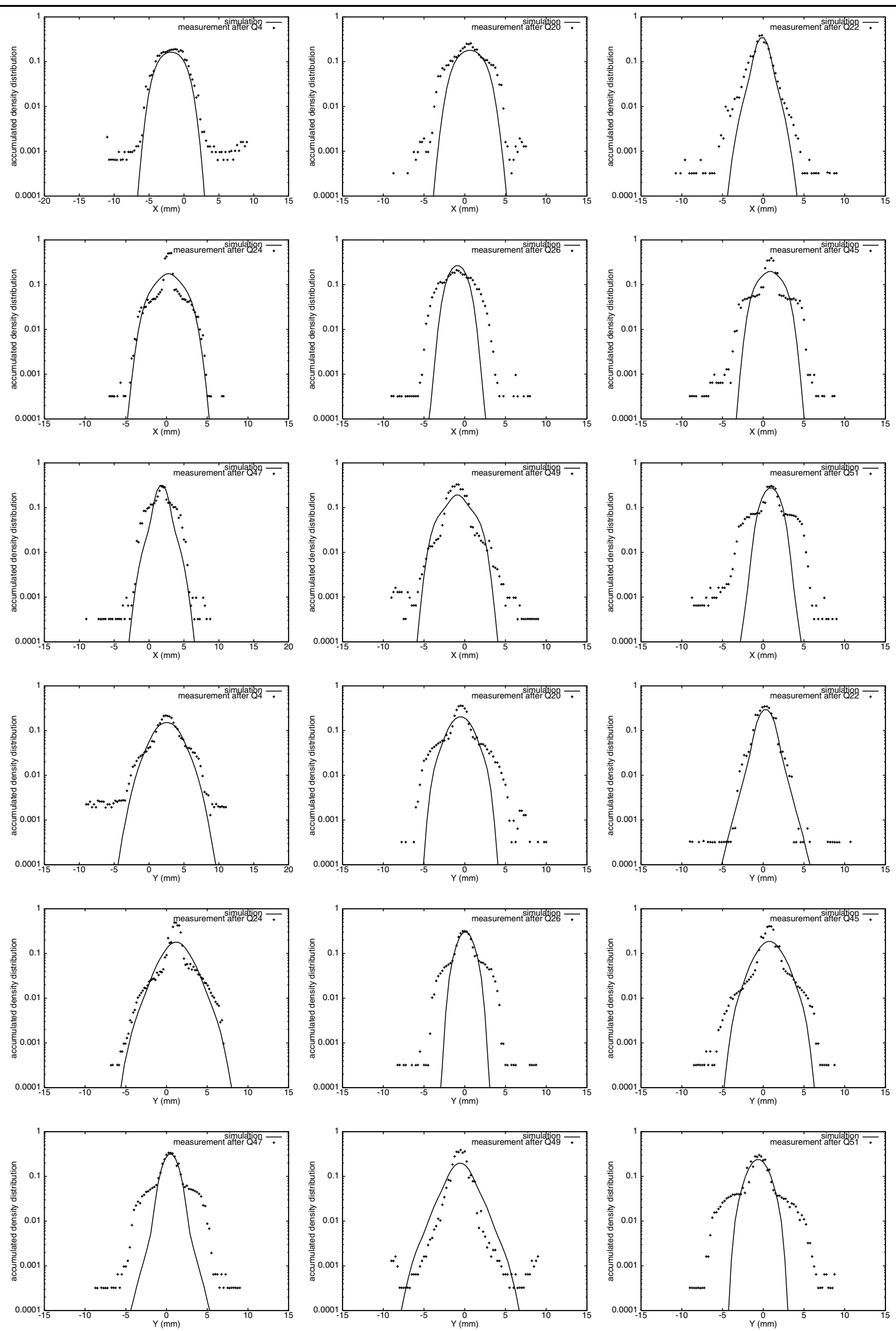

FIG. 8. $X$ and $y$ density distribution from simulation using initial Gaussian distribution and from measurements at $75 \mathrm{~mA}$ and mismatch factor of 1.5. The first nine plots correspond to the nine $x$ profiles and the second nine plots correspond to the nine $y$ profiles. 

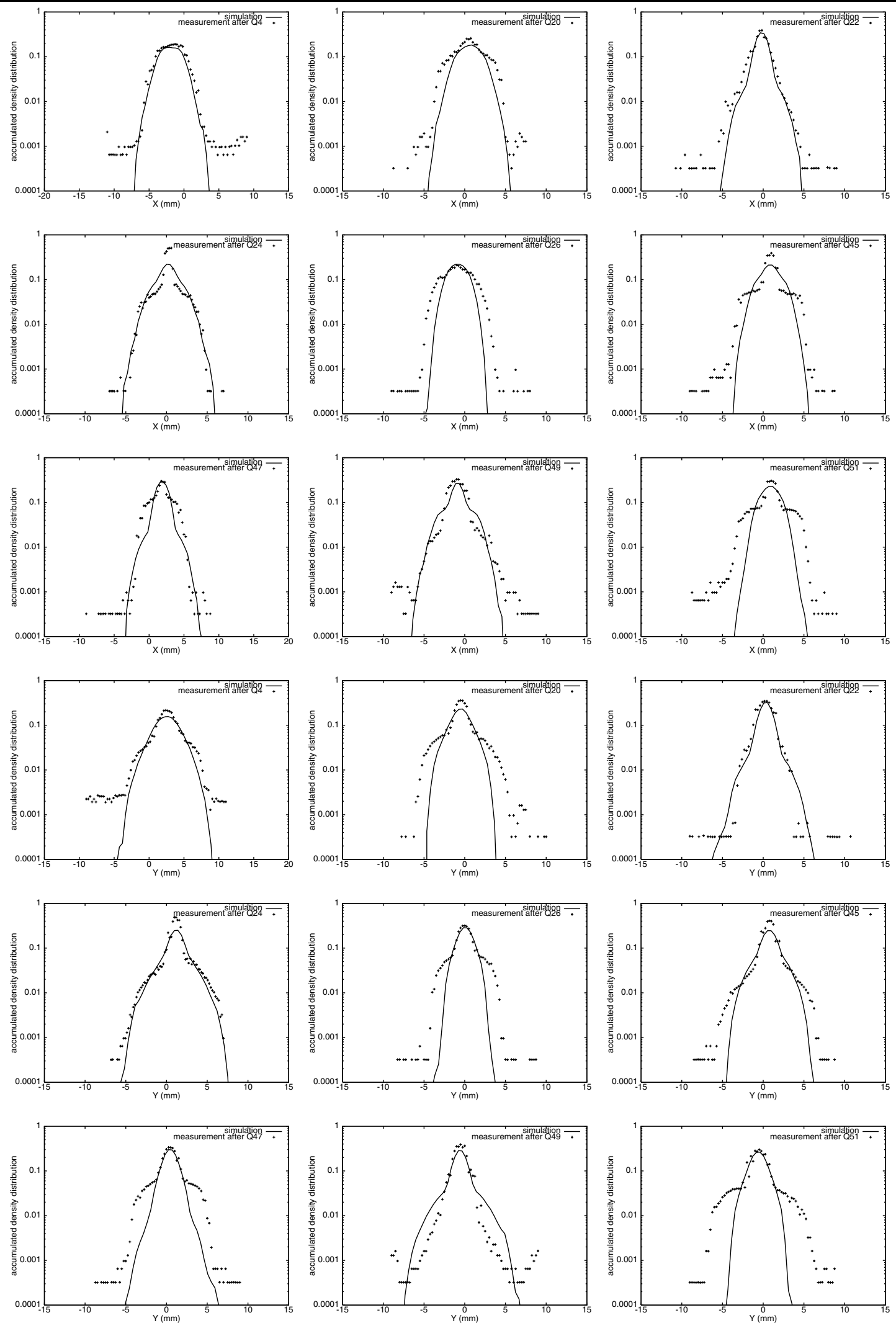

FIG. 9. $\quad X$ and $y$ density distribution from simulation using initial LEBT/RFQ output distribution and from measurements at $75 \mathrm{~mA}$ and mismatch factor of 1.5. The first nine plots correspond to the nine $x$ profiles and the second nine plots correspond to the nine $y$ profiles. 
the matched case shown in Figs. 4-6, the simulations using all three initial distributions fail to reproduce the broader shoulders of the experimental data. We interpret these broad shoulders as evidence of a rapid halo growth rate in the mismatched beam, which is not reproduced in the simulations.

Figure 10 compares the rms-emittance growth calculated from the measurements for $\mu=1.5$ with those from simulations. The emittance-growth rate calculated from the measurements is larger than that from simulations. We find that the emittance-growth rate from simulations increases as we progress from the Waterbag to Gaussian to LEBT/RFQ, i.e., with increasing halo population in the initial distribution. This is a result that would be expected qualitatively from the particle-core model [12], because the resonant particles that form the halo lie outside the beam core.

Figure 11 gives the emittances calculated from the measurements and from the simulations using the LEBT/RFQ initial distribution for a set of mismatch factors $1.0,1.25,1.5$, and 1.75 . We see that the simulations qualitatively reproduce the trend of increasing emittance as a function of mismatch factor. Quantitatively, the emittance-growth rate calculated from measurements is larger than that from simulations.

As a further test of the hypothesis that the emittance growth is enhanced by the population of the tail of the input beam, we have done simulations for $75 \mathrm{~mA}$ and a $\mu=1.5$ mismatch using a distribution that is the sum of two Gaussians with different relative heights and different rms sizes. Figure 12 shows the final emittance growth as a function of the relative heights of the two distributions for a fixed ratio of the rms widths equal to 4 . We see that as more particles populate the tail of the distribution, the final emittance growth increases. For a relative height of $20 \%$ for the two Gaussians, the averaged final emittance growth reaches a value of 1.46 , which is

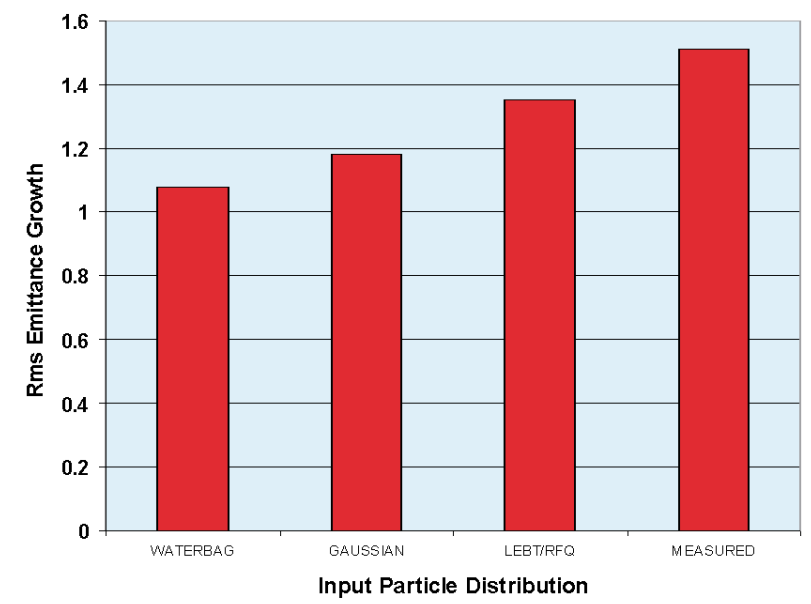

FIG. 10. (Color) A comparison of rms-emittance growth from simulations using three initial distributions with the measured experimental result for 1.5 breathing-mode mismatch.

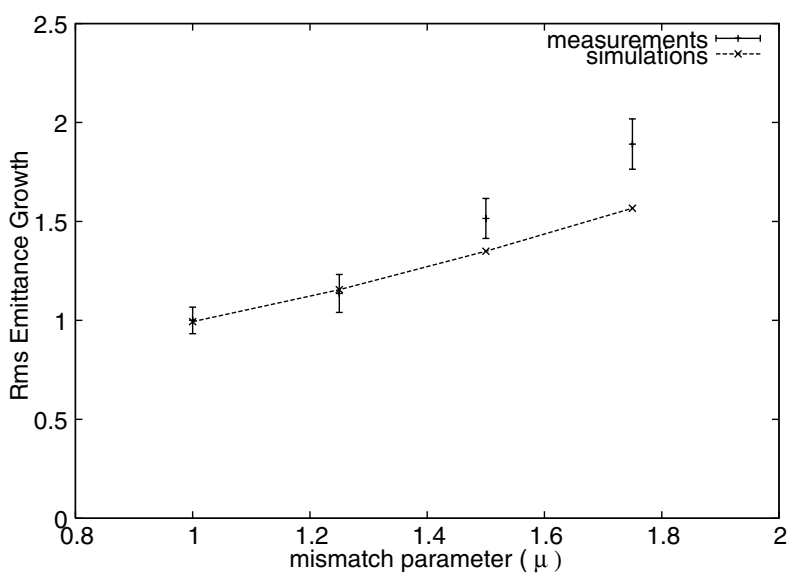

FIG. 11. Final rms-emittance growth from measurements (points with error bars) for breathing-mode mismatch at $75 \mathrm{~mA}$ as a function of mismatch parameter. Also shown (plots without error bars) are the simulation results for the initial LEBT/RFQ distribution.

within the experimental uncertainty of the measured value (1.51). This example confirms that an emittance growth of the approximate magnitude of the measured result can be obtained from simulation, by using an initial distribution with a large enough population in the tails. Figure 13 shows density profiles at the nine wire-scanner locations from the simulation using the initial double Gaussian with the $20 \%$ relative height, for comparison with the measurements. Broader shoulders have been generated using this initial distribution than for the previous three distributions, as expected. However, these simulation profiles still do not reproduce well the density profiles from the measurements. We believe that more experimental information about the initial distribution would be required to reproduce the details of the measured mismatched density profiles.

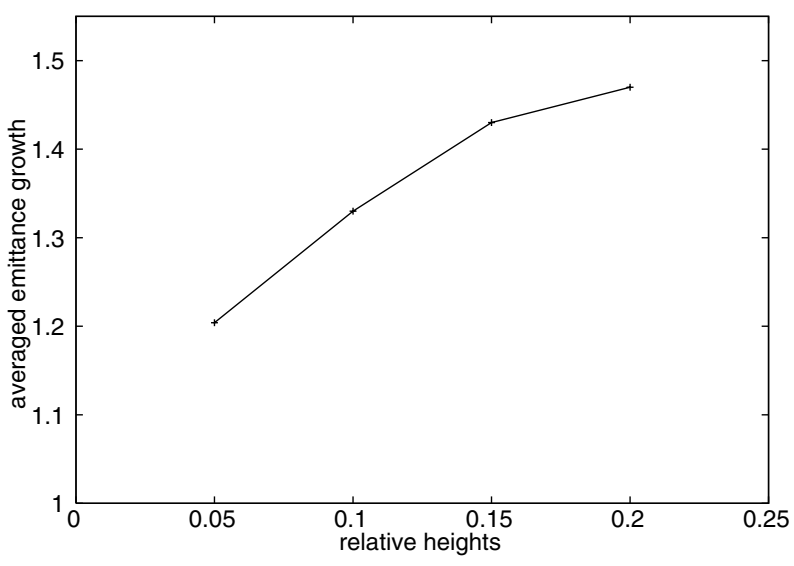

FIG. 12. Final rms-emittance growth for the doubleGaussian distribution as a function of the relative heights of the two Gaussian distributions for a fixed ratio of the rms widths equal to 4 . 

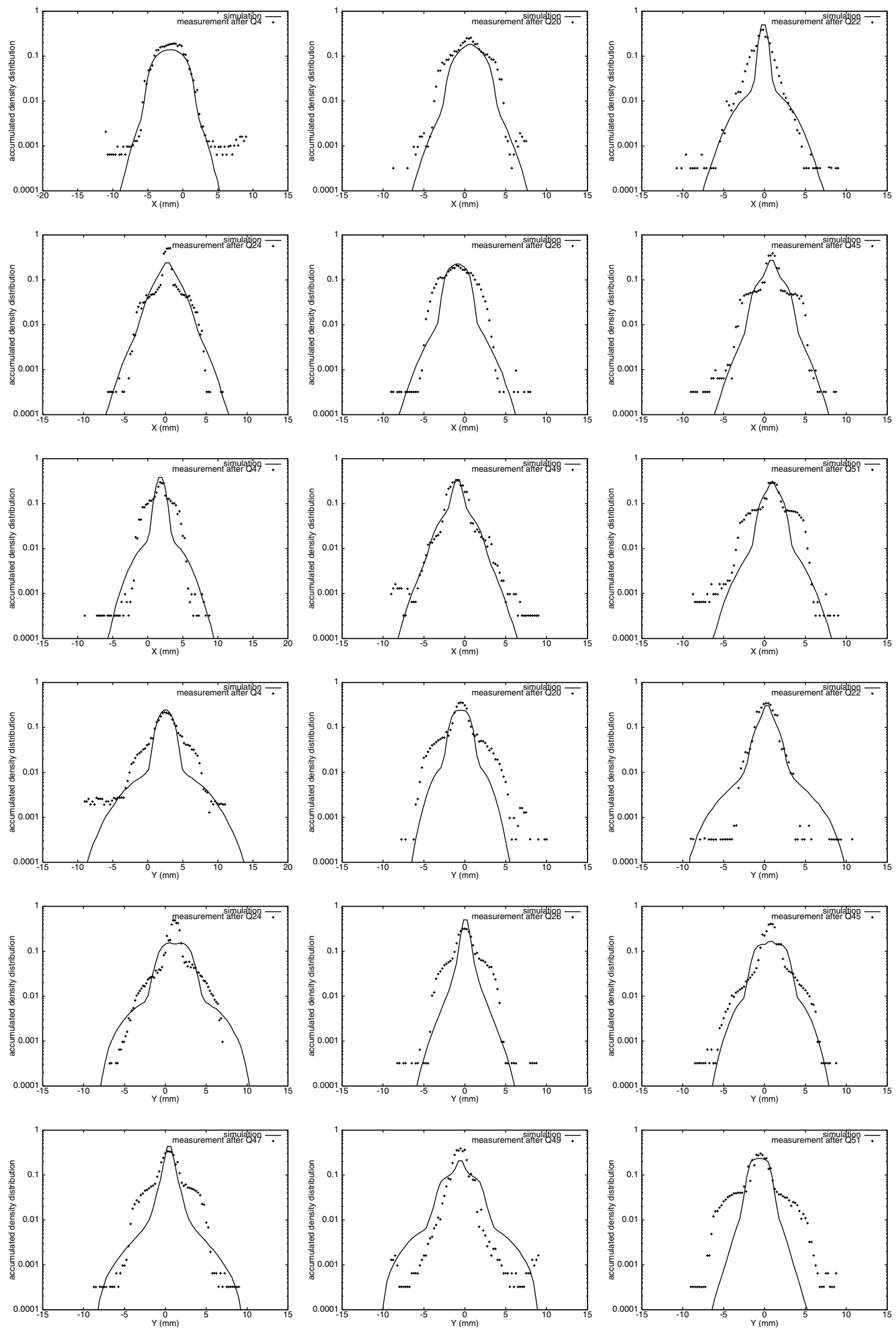

FIG. 13. $X$ and $y$ density distribution from simulation using initial double-Gaussian distribution and from measurements at $75 \mathrm{~mA}$ and mismatch factor of 1.5. The first nine plots correspond to the nine $x$ profiles and the second nine plots correspond to the nine $y$ profiles. 


\section{CONCLUSIONS}

Using the IMPACT code, we have carried out simulations of the LEDA beam halo experiment using four different initial particle distributions: 6D Waterbag, 6D Gaussian, LEBT/RFQ, and double Gaussian. All distributions have the same initial ellipse and emittance parameters, deduced from the measurements. The four distributions differ qualitatively with respect to their initial halo population. The measured mismatchedbeam profiles exhibit prominent shoulders, and a rapid emittance-growth rate. The 6D Waterbag, 6D Gaussian, and LEBT/RFQ distributions all underestimate this growth rate. Yet, the growth rate from simulations increases with increasing population of the tails of the input distribution, a result that is consistent with expectation from the particle-core model. A large population in the tails provided by the double-Gaussian distribution is successful in reproducing approximately the large measured emittance growth. Nevertheless, none of the initial distributions provides good agreement with the measured mismatched-beam profiles. We interpret that result as support for the hypothesis that the details of the mismatched-beam profiles are sensitive to the shape of the initial 6D distribution, which is not measured.

Benchmarking multiparticle simulation codes against experimental measurements is a challenge, especially when the details of the initial 6D phase-space distribution are not well known. This was the case for the LANL beam halo experiment. We have two major conclusions. (1) Using only the known Courant-Snyder parameters and the emittances as input parameters is not sufficient for reliable prediction of beam halo. The shape of the input distribution also matters. This conclusion is important because it is a common practice in accelerator design to simulate the beam-dynamics performance based on rms properties of the initial beam, without careful attention to the shape of the initial distribution. (2) The simulations show that higher emittance-growth rates for mismatched beams correspond to input distributions with a higher density in the initial tails. We interpret this result to be in accordance with the particle-core model, where one would expect that greater population of the tails means a greater population of the region of phase space that leads to resonant halo growth.

\section{ACKNOWLEDGMENTS}

We would like to thank Dr. L. Young for providing the initial distribution for the LEBT/RFQ simulation, Dr.
K. R. Crandall, Dr. R. Garnett, Dr. R. Ryne, and Dr. W. Lysenko for helpful discussions, and the LEDA team for their work to make the experiment possible. The simulation work was performed on the Cray T3E and IBM SP at the National Energy Research Scientific Computing Center located at Lawrence Berkeley National Laboratory, and the SGI Origin 2000 at the Advanced Computing Laboratory located at Los Alamos National Laboratory. The halo experiment was supported by the U.S. DOE and Office of Nuclear Energy, Science and Technology. The simulation was partly supported by the U.S. DOE, Office of Science, Division of High-Energy and Nuclear Physics, under the projects, Advanced Computing for 21st Century Accelerator Science and Technology, and the Los Alamos Accelerator Code Group.

[1] K. R. Crandall and T. P. Wangler, Los Alamos National Laboratory Report No. LA-UR-88-1546, 1988.

[2] A. Friedman, D. P. Grote, and I. Haber, Phys. Fluids B 4, 2203 (1992).

[3] H. Takeda and J. H. Billen, in Proceedings of the XIX International Linac Conference, Chicago, 1998 (Argonne National Laboratory, Chicago, 1998), pp. $156-158$.

[4] J. Qiang, R. D. Ryne, S. Habib, and V. Decyk, J. Comput. Phys. 163, 434 (2000).

[5] J. Qiang, R. D. Ryne, B. Blind, J. H. Billen, T. Bhatia, R.W. Garnett, G. Neuschaefer, and H. Takeda, Nucl. Instrum. Methods Phys. Res., Sect. A 457, 1 (2001).

[6] H. Vernon Smith, Jr., J. D. Schneider, and R. Sheffield, in Proceedings of the 2001 Particle Accelerator Conference, Chicago, IL, 2001 (IEEE, Piscataway, NJ, 2001), pp. 3296-3298.

[7] C. K. Allen et al., Phys. Rev. Lett. 89, 214802 (2002).

[8] W. P. Lysenko et al., in Proceedings of the XXI International Linac Conference, Gyeongju, Korea, 2002 (Pohang University of Science and Technology, Gyeongju, 2002).

[9] J. Qiang, R. D. Ryne, and R.W. Garnett, Phys. Rev. ST Accel. Beams 5, 064201 (2002).

[10] T. P. Wangler et al., in Proceedings of the 2001 Particle Accelerator Conference, Chicago, IL, 2001 (Ref. [6]), pp. 2923-2915; P. L. Colestock et al., ibid., pp. 170172; M. Schulze et al., ibid., pp. 591-593.

[11] K. R. Crandall and D. P. Rusthoi, Los Alamos National Laboratory Report No. LA-UR-90-4146, 1990.

[12] T. P. Wangler, K. R. Crandall, R. D. Ryne, and T. S. Wang, Phys. Rev. ST Accel. Beams 1, 084201 (1998). 